University of Puget Sound

Sound Ideas

All Faculty Scholarship

Faculty Scholarship

$11-1-1997$

\title{
Answer To Question \#55. Are There Pictorial Examples That Distinguish Covariant And Contravariant Vectors?
}

James C. Evans

University of Puget Sound, jcevans@pugetsound.edu

Follow this and additional works at: http://soundideas.pugetsound.edu/faculty_pubs

\section{Citation}

Evans, James C.. 1997. "Answer to Question \#55. Are there pictorial examples that distinguish covariant and contravariant vectors?." American Journal Of Physics 65(11): 1039-1039.

This Article is brought to you for free and open access by the Faculty Scholarship at Sound Ideas. It has been accepted for inclusion in All Faculty Scholarship by an authorized administrator of Sound Ideas. For more information, please contact soundideas@pugetsound.edu. 


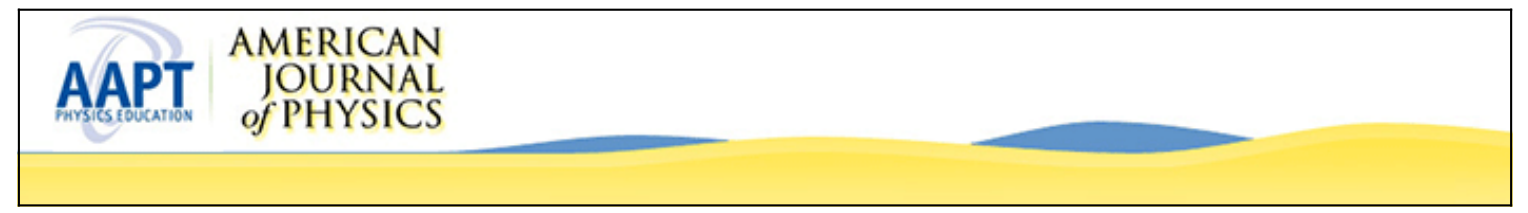

\section{Answer to Question \#55. Are there pictorial examples that distinguish covariant and contravariant vectors?}

James Evans

Citation: American Journal of Physics 65, 1039 (1997); doi: 10.1119/1.18736

View online: http://dx.doi.org/10.1119/1.18736

View Table of Contents: http://scitation.aip.org/content/aapt/journal/ajp/65/11 ?ver=pdfcov

Published by the American Association of Physics Teachers

\section{Articles you may be interested in}

Covariance of the total energy-momentum four-vector of a charge and current carrying macroscopic body Am. J. Phys. 66, 414 (1998); 10.1119/1.18879

Editorial Note on Answers to Question \#55. Are there pictorial examples that distinguish covariant and contravariant vectors?

Am. J. Phys. 65, 1037 (1997); 10.1119/1.18744

Answer to Question \#55. Are there pictorial examples that distinguish covariant and contravariant vectors? Am. J. Phys. 65, 1037 (1997); 10.1119/1.18743

Answer to Question \#55. Are there pictorial examples that distinguish covariant and contravariant vectors? Am. J. Phys. 65, 1038 (1997); 10.1119/1.18735

Question \#55. Are there pictorial examples that distinguish covariant and contravariant vectors?

Am. J. Phys. 65, 11 (1997); 10.1119/1.18492

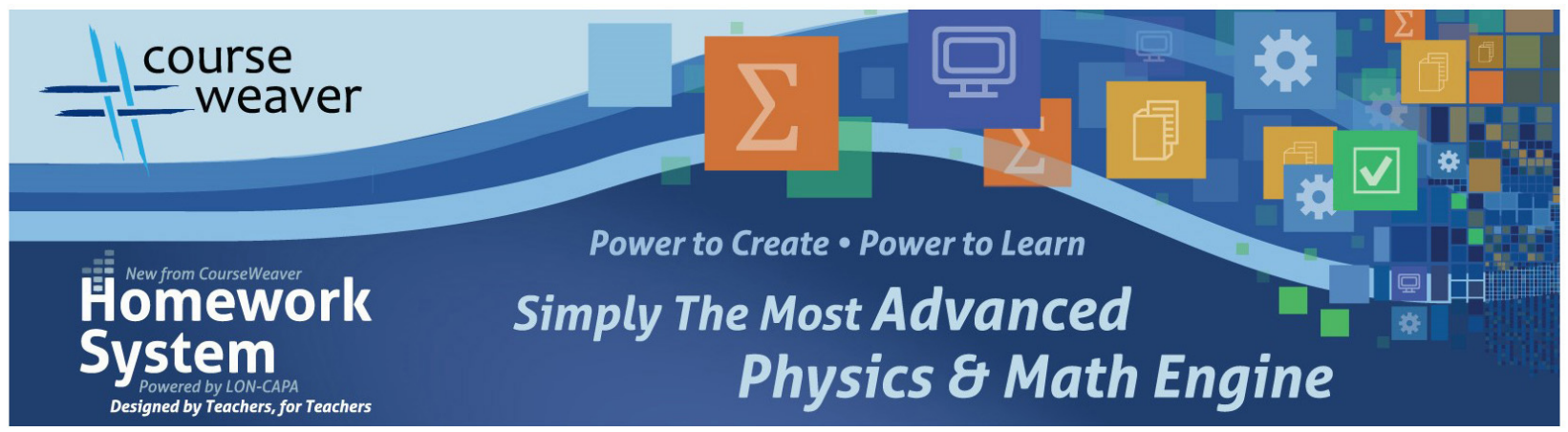




\section{Answer to Question \#55. Are there pictorial examples that distinguish covariant and contravariant vectors?}

It is important to distinguish ${ }^{1}$ between covariant and contravariant components of a vector whenever we deal with nondiagonal metric tensors, in fact, whenever the metric tensor is not the identity matrix. It is possible to construct informative pictorial examples even in the context of Euclidean plane geometry, if we choose to work with nonorthogonal axes.

Consider two axes, $x$ and $y$, inclined at an angle $\alpha$. An arbitrary vector $\mathbf{V}$ in the plane may be resolved into $x$ and $y$ components. But since the axes are not orthogonal, it is not so clear what we want to mean by "components of a vector." Two different definitions might be sensible. First, we might mean that $\mathbf{V}$ can be written in terms of line segments directed parallel to the unit vectors $\mathbf{x}$ and $\mathbf{y}$ :

$$
\mathbf{V}=V^{x} \mathbf{x}+V^{y} \mathbf{y} \text {. }
$$

$V^{x}$ and $V^{y}$ are the contravariant components of $\mathbf{V}$. Second, we might mean that we should be able to pick off components by taking inner products of $\mathbf{V}$ with $\mathbf{x}$ and $\mathbf{y}$ :

$$
V_{x}=\mathbf{V} \cdot \mathbf{x}=V \cos \theta_{x}, \quad V_{y}=\mathbf{V} \cdot \mathbf{y}=V \cos \theta_{y}
$$

(where $\theta_{x}$ and $\theta_{y}$ are the angles $\mathbf{V}$ makes with the $x$ and $y$ axes). $V_{x}$ and $V_{y}$ are the covariant components of $\mathbf{V} . V^{x}, V^{y}$ and $V_{x}, V_{y}$ are shown in diagram 1.

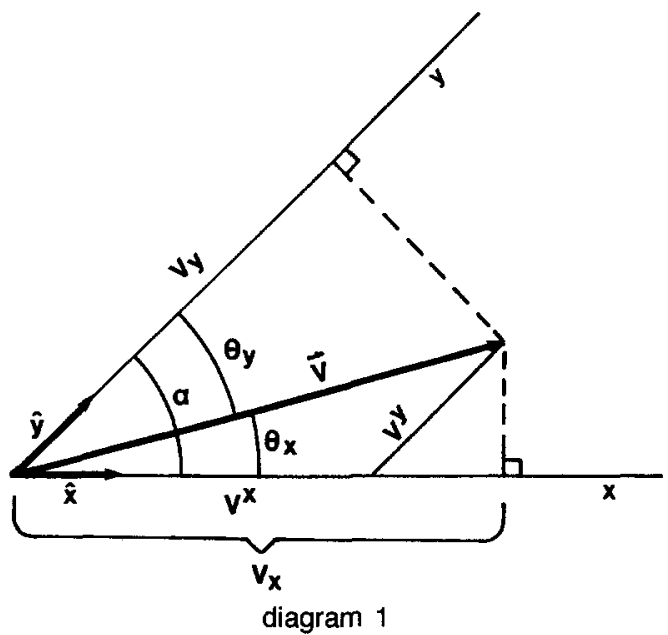

Let us construct the metric tensor. Taking the inner product of $\mathbf{x}$ with Eq. (1) we have

$$
V_{x}=V^{x}+V^{y} \cos \alpha,
$$

since $\mathbf{x} \cdot \mathbf{y}=\cos \alpha$. Similarly, taking the inner product of $\mathbf{y}$ with Eq. (1) gives

$$
V_{y}=V^{x} \cos \alpha+V^{y} .
$$

Thus the covariant components may be obtained from the contravariant components by the rule

$$
V_{i}=g_{i j} V^{j},
$$

where $i$ and $j$ can each take on the values $x$ and $y$, and where the repeated index is summed. The elements of the metric tensor are then

$$
g_{i j}=\left(\begin{array}{cc}
1 & \cos \alpha \\
\cos \alpha & 1
\end{array}\right) \text {. }
$$

It is easy to show that the inverse transformation is

$$
V^{i}=g^{i j} V_{j},
$$

with

$$
g^{i j}=\left(\begin{array}{cc}
\sin ^{-2} \alpha & -\cos \alpha \sin ^{-2} \alpha \\
-\cos \alpha \sin ^{-2} \alpha & \sin ^{-2} \alpha
\end{array}\right) .
$$

The metric tensor has the usual properties. For example, $V^{2}=\mathbf{V} \cdot \mathbf{V}$ is an invariant, independent of the angle $\alpha$ between the axes. It may be calculated from

$$
V^{2}=V^{i} V_{i}=V^{i} g_{i j} V^{j}=V_{i} g^{i j} V_{j} .
$$

An easy way to see this is to begin from diagram 2,

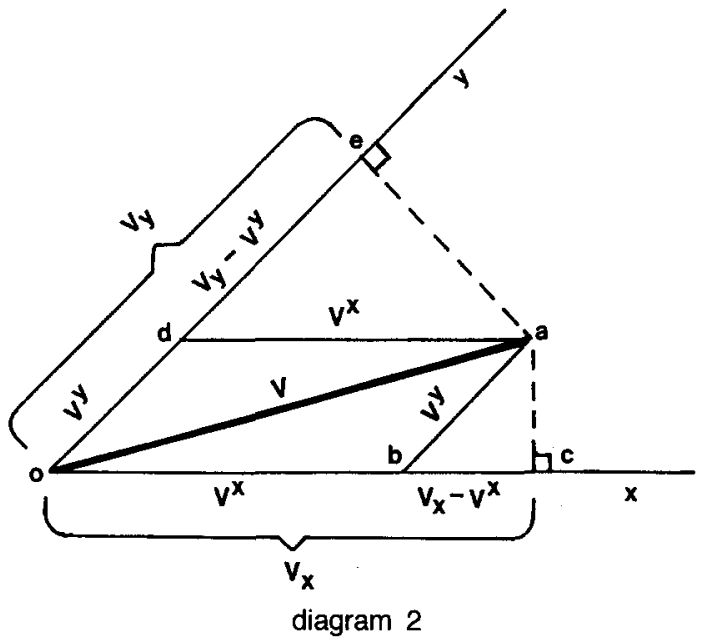

which is merely a more symmetric version of diagram 1 . For the sake of having an expression of symmetric form, let us write

$$
V^{2}=\frac{1}{2}\left[(o c)^{2}+(c a)^{2}+(o e)^{2}+(e a)^{2}\right] .
$$

Now,

$$
\begin{aligned}
& o c=V_{x} \\
& (c a)^{2}=(a b)^{2}-(b c)^{2}=\left(V^{y}\right)^{2}-\left(V_{x}-V^{x}\right)^{2} \\
& o e=V_{y} \\
& (e a)^{2}=(a d)^{2}-(d e)^{2}=\left(V^{x}\right)^{2}-\left(V_{y}-V^{y}\right)^{2} .
\end{aligned}
$$

With these substitutions, we find immediately

$$
V^{2}=V^{x} V_{x}+V^{y} V_{y} .
$$

Similarly, if $\mathbf{A}$ and $\mathbf{B}$ are two vectors, it may be shown that the invariant inner product (independent of $\alpha$ ) is given by

$$
\mathbf{A} \cdot \mathbf{B}=A B \cos \theta_{A B}=A^{i} B_{i}=A_{i} B^{i},
$$

where $\theta_{A B}$ is the angle between $\mathbf{A}$ and $\mathbf{B}$.

${ }^{1}$ D. Neuenschwander, Am. J. Phys. 65(1), 11 (1997).

James Evans Department of Physics University of Puget Sound Tacoma, Washington 98416 JCEvans@UPS.edu 